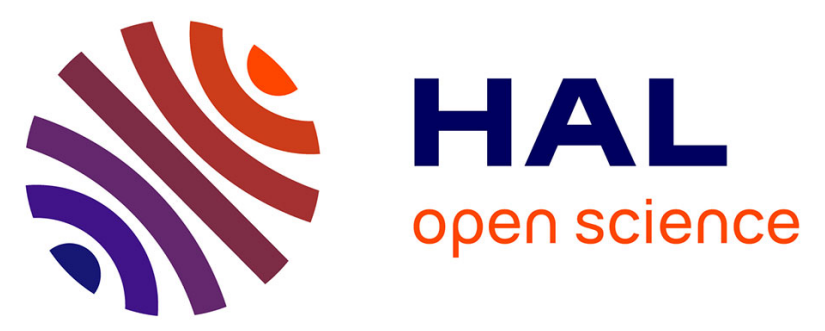

\title{
Introduction of shear warping in fibre beam elements for the computation of the nonlinear behaviour of concrete beams
}

\author{
Sophie Capdevielle, Stéphane Grange, Frédéric Dufour, C. Desprez
}

\section{To cite this version:}

Sophie Capdevielle, Stéphane Grange, Frédéric Dufour, C. Desprez. Introduction of shear warping in fibre beam elements for the computation of the nonlinear behaviour of concrete beams. EURO-C, Computational Modelling of Concrete and Concrete Structures, Feb 2018, Bad Hofgastein, Austria. hal-01836422

\section{HAL Id: hal-01836422 \\ https://hal.science/hal-01836422}

Submitted on 12 Jul 2018

HAL is a multi-disciplinary open access archive for the deposit and dissemination of scientific research documents, whether they are published or not. The documents may come from teaching and research institutions in France or abroad, or from public or private research centers.
L'archive ouverte pluridisciplinaire HAL, est destinée au dépôt et à la diffusion de documents scientifiques de niveau recherche, publiés ou non, émanant des établissements d'enseignement et de recherche français ou étrangers, des laboratoires publics ou privés. 


\title{
Introduction of shear warping in fibre beam elements for the computation of the nonlinear behaviour of concrete beams
}

\author{
S. Capdevielle \\ LMT, ENS Cachan, CNRS, Université Paris-Saclay, 94235 Cachan, France.
}

S. Grange

Univ Lyon, INSA-Lyon, GEOMAS, F-69621, France.

F. Dufour

Univ. Grenoble Alpes, CNRS, Grenoble INP, 3SR, F-38000 Grenoble, France.

C. Desprez

Université Paris-Est, MAST, SDOA, IFSTTAR, F-77447 Marne-la-Vallée, France.

\begin{abstract}
The present paper deals with modelling the nonlinear behaviour of concrete using the fibre element method. Proved to be efficient for slender structural elements mainly subject to normal stresses, the method lacks in accuracy when modelling the effect of shear. The present contribution proposes to enhanced a Timoshenko fibre beam element by adding shear warping deformations in the formulation. Transverse shear as well as torsion warping is taken into account, enabling the computation of the response to a complex loading. Warping degrees of freedom are computed simultaneously with the beam degrees of freedom by a fully implicit solution procedure. Concrete is modelled using a 3D damage model. Due to the coupling of warping and global beam degrees of freedom, the warping displacements in the beam element can evolve with the evolution of concrete damage. The beam element enhancement is validated at the cross-section scale considering a linear elastic behaviour. Enhanced beam elements are then used to model experimentally-tested structural elements. Both the linear and non-linear parts of the structural response are investigated.
\end{abstract}

\section{INTRODUCTION}

Modelling a structure using fibre beam elements is proved to be an efficient approach (Grange et al. 2008, Kotronis and Mazars 2005, Kotronis and Grange 2010). The method enables the modeller to carry out computations at the structural scale, taking into account the nonlinear behaviour of concrete, at a relatively low computational cost. Based on beam elements, the fibre beam element approach avoids the use of a generalized constitutive law by adding the crosssection scale. From the generalized strains, strains in the cross section are computed according to a kinematics assumption. Stresses are obtained from the strains at each integration point of the cross section by a chosen constitutive law. By integrating the stresses over the cross-section, generalized forces are eventually computed (Spacone and El-Tawil 2004). The results are convincing for structural elements subject to normal stresses. However, the computed behaviour lacks in accuracy in the case of large shear stresses.
Indeed, the plane cross-section assumption used to compute the strains is not precise enough to convey the effect of shear.

Several authors have proposed to address this problem by introducing warping in the kinematics of fibre beam elements (Mazars et al. 2006, Bairan and Mari 2007, Le Corvec and Filippou 2011, Capdevielle et al. 2016). Based on these approaches, an improved fibre beam element is developed in the present paper. Warping degrees of freedom, accounting for both torsion and shear warping are introduced in the formulation. The warping degrees of freedom are computed along with the global beam degrees of freedom by satisfying the structure equilibrium through an implicit solution procedure. The concrete behaviour is represented by the $\mathrm{Mu}$ model (Mazars et al. 2014). Through the nonlinear solution procedure, warping evolution is fully coupled with the damage evolution in the cross section. 
After a summary of the main equations of the enhanced element formulation, the numerical behaviour of the beam element is investigated at the crosssection scale. The behaviour of a cantilever beam subject to a vertical end-load is computed using a linear elastic material behaviour. The warping strains in the beam are compared to the analytical solution. To investigate the effect of warping coupled with the material nonlinearities, the response of a reinforced concrete structure subject to a dynamic load is eventually simulated.

\section{FORMULATION OF THE ENHANCED ELEMENT}

A 3D displacement-based Timoshenko fibre beam element is enhanced by the addition of warping degrees of freedom. This part presents the main equations of the enhancement. The modified kinematics is described first, followed by the variational formulation of the equilibrium equations. Some specificities of the shape functions required by the enhancement are eventually presented.

\subsection{Enhancement of the kinematics}

The vector $\mathbf{u}$ of displacements in the beam is additively decomposed into a plane displacement part $\mathbf{u}^{p}$ and a part coming from the cross-section warping $\mathbf{u}^{w}$. The two parts are assumed to be orthogonal (Bairan and Mari 2006), which means that warping displacements are free from any rigid body motion of the cross-section. $\mathbf{u}^{p}$ contains the classical displacements in Timoshenko's cross section. Warping displacements are assumed to occur only in the longitudinal $x$ direction. $u, v$ and $w$ represent the mean translations of the cross section, respectively in the $x, y$ and $z$ directions. $\theta_{x}, \theta_{x}$ and $\theta_{x}$ are the mean rotations.

$\mathbf{u}=\mathbf{u}^{p}+\mathbf{u}^{w}=\left[\begin{array}{c}u-y \theta_{z}+z \theta_{y} \\ v-z \theta_{x} \\ w+y \theta_{x}\end{array}\right]+\left[\begin{array}{c}u_{x}^{w}(x, y, z) \\ 0 \\ 0\end{array}\right]$

From the displacements described in equation 1, the linearized strain tensor $\varepsilon$ can be computed as follows:

$\varepsilon=\frac{1}{2}\left(\operatorname{grad}(\mathbf{u})+\operatorname{grad}(\mathbf{u})^{T}\right)=\varepsilon^{p}+\varepsilon^{w}$

The strain vector $\epsilon$ used in the beam formulation is thus computed as described in equation 3 .

$$
\boldsymbol{\epsilon}=\left[\begin{array}{c}
\epsilon_{x x} \\
2 \epsilon_{x y} \\
2 \epsilon_{x z}
\end{array}\right]=\underbrace{\left[\begin{array}{c}
\frac{d u}{d x}-y \frac{d \theta_{z}}{d x}+z \frac{d \theta_{y}}{d x} \\
-z \frac{d \theta_{x}}{d x}+\frac{d v}{d x}-\theta_{z} \\
y \frac{d \theta_{x}}{d x}+\frac{d w}{d x}+\theta_{y}
\end{array}\right]}_{\boldsymbol{\epsilon}^{p}}+\underbrace{\left[\begin{array}{c}
\frac{\partial u_{x}^{w}}{\partial x} \\
\frac{\partial u_{x}^{w}}{\partial y} \\
\frac{\partial u_{x}^{w}}{\partial z}
\end{array}\right]}_{\boldsymbol{\epsilon}^{w}}
$$

\subsection{Variational form of the equilibrium equation}

Assuming that there is no body force, the variational form of the beam equilibrium equation is given in equation 4. $\sigma$ denotes Cauchy's stress tensor and $\delta \mathbf{u}$ the virtual displacements. $\Omega$ represents the beam integration domain.

$\int_{\Omega} \delta \mathbf{u}^{T} \operatorname{div}(\boldsymbol{\sigma}) d \Omega=0$

The virtual displacements are assumed to be decomposed in a plane-section part and a warping part, like the real displacement field.

$\int_{\Omega}\left(\delta \mathbf{u}^{p T}+\delta \mathbf{u}^{w T}\right) \operatorname{div}(\boldsymbol{\sigma}) d \Omega=0$

Taking into account the orthogonality of the planesection displacement field and the warping displacement field, equation 5 is projected on the corresponding subspaces. This leads to two sets of equations (6). By integrating by part each integral in equation 6 , we get the final weak form of the equilibrium equations (7).

$$
\left\{\begin{array}{l}
\int_{\Omega} \delta \mathbf{u}^{p T} \operatorname{div}(\boldsymbol{\sigma}) d \Omega=0 \\
\int_{\Omega} \delta \mathbf{u}^{w T} \operatorname{div}(\boldsymbol{\sigma}) d \Omega=0
\end{array}\right.
$$

$\left\{\begin{array}{l}\int_{\Omega} \delta \boldsymbol{\epsilon}^{p T} \boldsymbol{\sigma} d \Omega=\int_{\partial \Omega} \delta \mathbf{u}^{p T}(\boldsymbol{\sigma} \mathbf{n}) d S \\ \int_{\Omega} \delta \boldsymbol{\epsilon}^{w T} \boldsymbol{\sigma} d \Omega=\int_{\partial \Omega} \delta \mathbf{u}_{x}^{w T}\left(\sigma_{x x} n_{x}\right) d S\end{array}\right.$

\subsection{Beam finite element formulation}

A Timoshenko beam element with linear longitudinal shape functions is enhanced. In the transverse directions, the cross-section is discretized using linear triangles.

To enforce the orthogonality of plane section and warping displacements, rigid-body motions of the cross section need to be removed from the shape functions used to interpolate warping displacements. The 
transverse shape functions $\boldsymbol{N}_{i}$ are thus projected on the subspace orthogonal to the plane displacement field (Le Corvec 2012). The resulting shape functions $\tilde{\mathbf{N}}_{i}$, given in equation 8 are used for warping displacements. The derivatives of the projected shape functions (equation 10) also need to be computed for the beam element formulation (Capdevielle et al. 2017).

$$
\tilde{\boldsymbol{N}}_{i}=\boldsymbol{N}_{i}-\int_{\mathcal{S}} \boldsymbol{N}_{i}\left[\begin{array}{lll}
\frac{1}{a_{1}} & \frac{y}{a_{2}} & \frac{z}{a_{3}}
\end{array}\right] d \mathcal{S}\left[\begin{array}{l}
1 \\
y \\
z
\end{array}\right]
$$

Where $\mathcal{S}$ represents the cross-section integration domain, and $a_{1}, a_{2}$ and $a_{3}$ are computed as follows:

$$
\begin{aligned}
& a_{1}=\int_{\mathcal{S}} 1 d \mathcal{S} \\
& a_{2}=\int_{\mathcal{S}} y^{2} d \mathcal{S} \\
& a_{3}=\int_{\mathcal{S}} z^{2} d \mathcal{S} \\
& \left\{\begin{array}{c}
\frac{\partial \tilde{\mathbf{N}}_{i}}{\partial y}=\frac{\partial \boldsymbol{N}_{i}}{\partial y}-\int_{\mathcal{S}} \boldsymbol{N}_{i} \frac{y}{a_{2}} d \mathcal{S} \\
\frac{\partial \tilde{\mathbf{N}}_{i}}{\partial z}=\frac{\partial \boldsymbol{N}_{i}}{\partial z}-\int_{\mathcal{S}} \boldsymbol{N}_{i} \frac{z}{a_{3}} d \mathcal{S}
\end{array}\right.
\end{aligned}
$$

In the longitudinal direction, the warping degrees of freedom of each cross section are interpolated to the nodes of the beam element using the beam shape functions.

\subsection{Solution procedure}

Let the matrices $\boldsymbol{B}_{p}$ and $\boldsymbol{B}_{w}$ contain the derivatives of the longitudinal shape functions, and the matrices $\mathbf{a}_{s}$ and $\mathbf{a}_{w}$ the transverse shape functions and there derivatives. After discretisation of the variational form of the equilibrium equations (7), we obtain the following non-linear system of equations to solve:

$$
\boldsymbol{A}_{e=1}^{n_{e l}} \underbrace{\left[\begin{array}{l}
\int_{\Omega^{e}} \boldsymbol{B}_{p}^{T} \mathbf{a}_{s}^{T} \hat{\boldsymbol{\sigma}}\left(\boldsymbol{\epsilon}^{p}, \boldsymbol{\epsilon}^{w}\right) d \Omega^{e} \\
\int_{\Omega^{e}} \boldsymbol{B}_{w}^{T} \mathbf{a}_{w}{ }^{T} \hat{\boldsymbol{\sigma}}\left(\boldsymbol{\epsilon}^{p}, \boldsymbol{\epsilon}^{w}\right) d \Omega^{e}
\end{array}\right]}_{\mathbf{P}_{\mathrm{el}}}=\left[\begin{array}{c}
\boldsymbol{F}_{\mathrm{ext}} \\
\boldsymbol{F}^{w}
\end{array}\right]
$$

In equation $11, \boldsymbol{F}_{\text {ext }}$ represents the external forces. $\boldsymbol{F}^{w}$ are the forces due to restrained warping boundary conditions. The longitudinal interpolation of warping degrees of freedom enables to take into account a variation of warping due to the boundary conditions in the longitudinal direction. The warping nodal degrees of freedom are added to the global beam degrees of freedom in the final system of equations.

The nonlinear system of equations (11) is solved using a fully nonlinear solution procedure based on Newmark's method. The enhanced element is implemented in the finite element code ATLAS (Grange 2015). This code is used to compute the numerical examples presented in the next paragraphs.

\section{WARPING PROFILE AT THE CROSS-SECTION SCALE}

The enhanced fibre beam element built in the previous part is tested here under transverse shear. The case study is presented in figure 1 . A cantilever beam of $1 \mathrm{~m}$ length is subject to a vertical load of $1 \mathrm{~N}$ applied at its free end. The cross-section is rectangular with dimensions $0.1 \mathrm{~m} \times 0.2 \mathrm{~m}$. The beam is discretized in 10 enhanced fibre elements. This relatively high number of beam elements is chosen to reduce the error linked to the longitudinal interpolation with linear shape functions. Two cross-section meshes are used, displayed in figure 2. A linear elastic material is considered, with a Young's modulus of $30 \mathrm{GPa}$ and a Poisson's ratio of 0.2 .

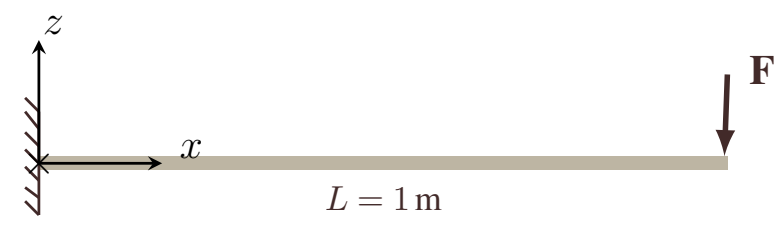

Figure 1: Considered case study for the analytical validation of the enhanced fibre beam element in transverse shear

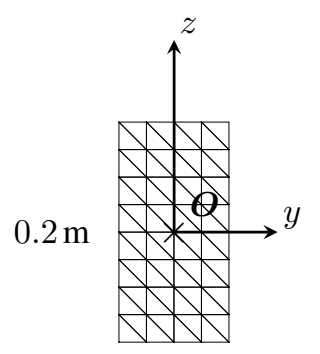

$0.1 \mathrm{~m}$

Mesh 1

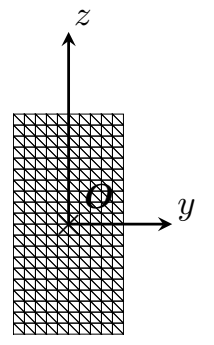

$0.1 \mathrm{~m}$
Figure 2: Cross-section meshes used for the analytical validation of the enhanced fibre beam element.

The strains obtained with the enhanced fibre beam computation are displayed in figure 3 . They are compared to the analytical strains obtained by Jourawski's theory. These results are extracted from (Capdevielle et al. 2017). They show that the linear elastic strains accounting for transverse shear warping are well computed by the enhanced fibre beam element. 


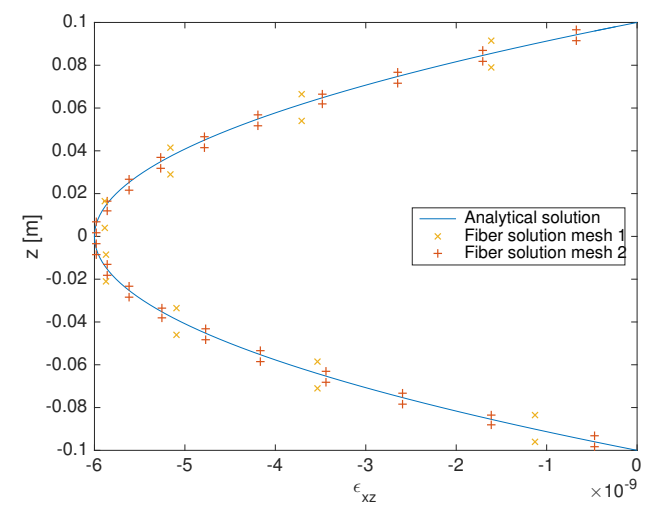

Figure 3: Results for the bended cantilever beam case-study: comparison of the computed shear strains to the analytical shear strains for the bended cantilever beam.

Since the warping degrees of freedom are linked at the nodes through the longitudinal interpolation, it is possible to take into account the effect of restrained warping at the boundary conditions. The results of the cantilever beam case study are further investigated here. Warping is assumed to be prevented at the fixed end. Figure 4 displays the warping displacements in both the cross-sections closest and furthest to the fixed boundary condition.

The warping displacements are greater close to the free end of the beam than close to the fixed boundary condition (see figure 4). This result confirms the fact that the longitudinal interpolation of warping displacements enables the solution to account for constrained warping conditions.

More results on the linear elastic validation of the transverse shear and coupled shear/torsion behaviour are presented in (Capdevielle et al. 2017).

\section{INFLUENCE OF WARPING ON THE STRUCTURAL DYNAMIC BEHAVIOUR}

Once the linear elastic behaviour of the enhanced beam element is validated, the effect of warping at the structural scale with a damaging material is investigated. The CAMUS mock-up (CEA 1998), shown in figure 5(a) is studied here. It is a reinforced concrete structure, representing a 5-story building. It comprises two walls of section $1,70 \mathrm{~m} \times 0,06 \mathrm{~m}$. The walls are connected by reinforced concrete slabs representing the floors and supporting the added weight. Each story is $0.90 \mathrm{~m} \mathrm{high}$, and the basement is $0,6 \mathrm{~m}$ high. The mock-up is fixed on a shaking table. Seismic signals of increasing intensity have been applied to the structure in the direction of the wall plane.

The study aims at investigating the effect of including warping in the formulation on the numerical response of the structure. The fibre beam element model used here is based on (De Biasio 2014). Each

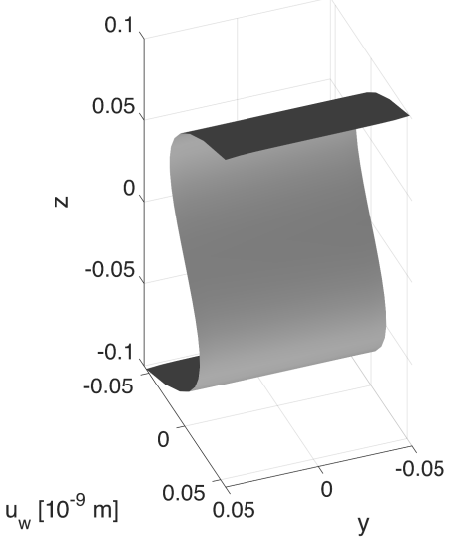

(a)

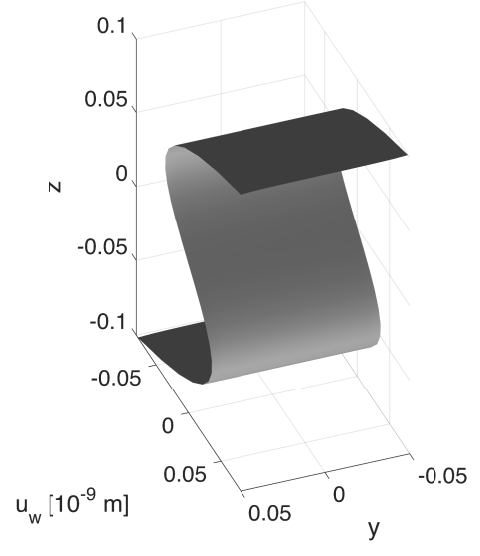

(b)

Figure 4: Warping displacements in different cross-sections of the beam displayed in figure 1. 4(a): Closest cross-section to the fixed boudary conditions. 4(b): Closest cross-section to the beam's free end.

story is discretized into 3 fibre beam elements. The floor mass is lumped at the corresponding nodes. The cross-section mesh is displayed in figure 4 . Each wall is discretised into 12 triangular elements. A simplified mesh for the steel rebars is used, with 3 triangular elements in each wall.

The first fundamental frequencies of the structure are computed, without and with the warping enhancement in the fibre elements. The shaking table is not modelled here. The results are given in table 1.

\begin{tabular}{|c|ccc|}
\hline Frequencies [Hz] & Mode 1 & Mode 2 & Mode 3 \\
\hline Without warping & 9.45 & 44.55 & 46.11 \\
With warping & 9.01 & 43.29 & 43.87 \\
\hline Relative difference & $5 \%$ & $3 \%$ & $5 \%$ \\
\hline
\end{tabular}

Table 1: First fundamental frequencies of the CAMUS structure obtained by a numerical modal analysis without modelling the shaking table 


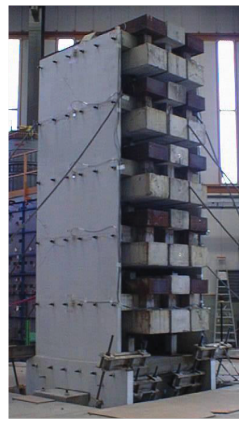

(a)

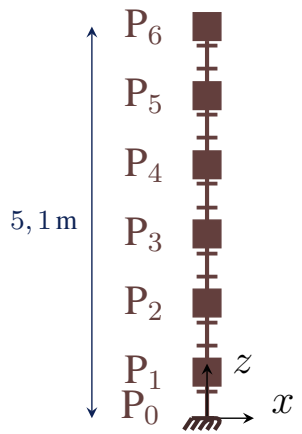

(b)
Figure 5: CAMUS mock-up (De Biasio 2014) and fibre element numerical model.

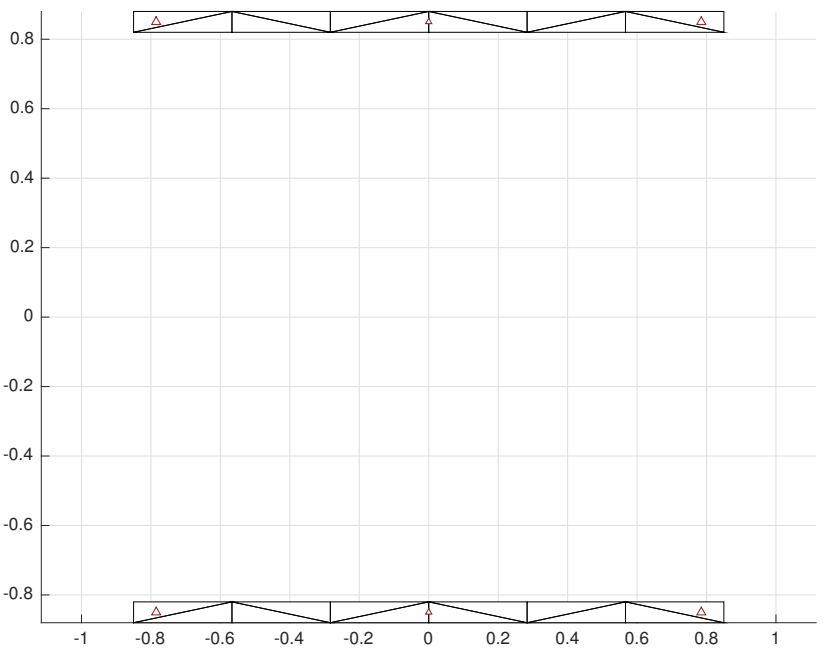

Figure 6: Mesh of the cross-section.

The fundamental frequencies corresponding to flexural modes are shifted by $5 \%$ by adding warping in the model. The model without warping is stiffer. This is consistent with results obtained in torsion, for which adding warping in the model reduces the initial stiffness of the torque-twist curve (Mazars et al. 2006, Capdevielle et al. 2016).

A dynamic time-analysis of the CAMUS structure is then carried out. Concrete is modelled using the $\mathrm{Mu}$ damage model (Mazars et al. 2014). The steel rebars are modelled using the Menegott Pinto model (Menegotto and Pinto 1973). The accelerogram applied to the structure is given in figure 7. A Rayleigh damping of $2 \%$ is considered in the model.

The resulting top displacements of the structure, respectively computed without and with the warping enhancement, are given in figure 8 . It can be seen that the difference in the fundamental frequencies induces a progressive shifting of the two response curves. It can also be noted that the model without warping results in a residual displacement at the end of the computation, whereas there is no residual displacement

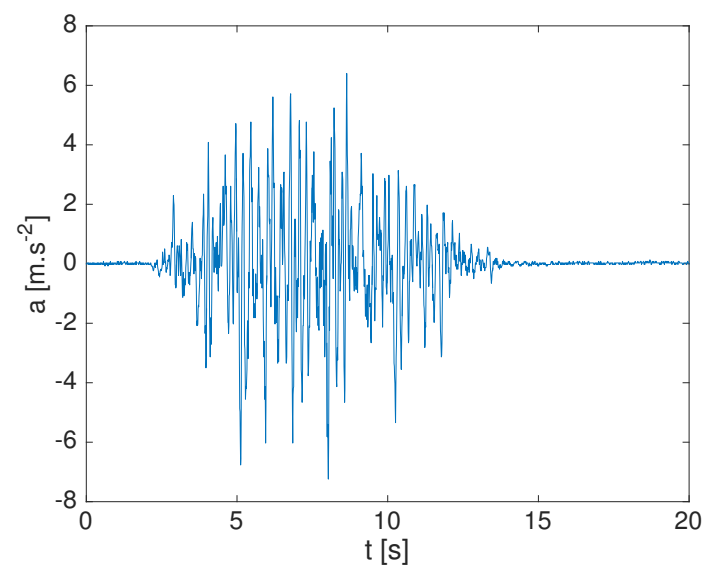

Figure 7: Accelerogram of the signal applied to the CAMUS structure

for the model with warping. This is probably due to the yielding of the reinforcing rebars in the case of the model without warping. These results need more investigation before a definitive conclusion can be assessed.

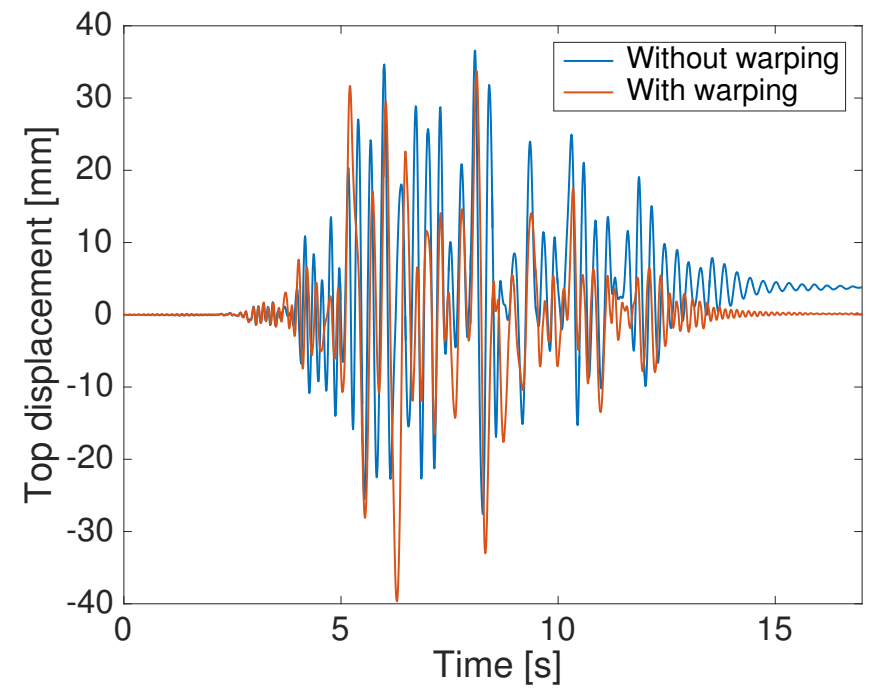

Figure 8: Top displacement of the mock-up during the seismic loading; comparison of the numerical results using the enhanced element without and with warping.

This case-study clearly demonstrates the effect of warping on the structural behaviour. Though, it does not show the importance of including warping in the formulation to represent the real behaviour of a structure, even if accounting for warping is physically relevant. Indeed, by suitably calibrating the shaking table model, both models without and with warping are able to represent the experimental behaviour. A further study needs to be conducted to assess the importance of taking warping into account. A set of beams experimentally tested in torsion has been modelled with the enhanced element and will be presented at the conference. 


\section{CONCLUDING REMARKS}

An enhanced fibre beam element with additional warping degrees of freedom has been presented. The enhancement is formulated by adding warping displacements to the kinematics of a Timoshenko fibre beam element. The warping shape functions to interpolate warping displacements in the transverse directions are set to be free of the rigid-body motions of the cross-section. A first numerical example has been presented on a cantilever beam subject to a vertical load. With a linear elastic material, an analytical solution is available. The numerically computed strains have been successfully compared to the analytical strains in the cross section. Then, the enhanced element has been used to model a structure under dynamic loading. Although the difference in fundamental frequencies between the model without and with the warping enhancement is small, it induces a remarkable difference in the nonlinear dynamic response of the structure. This study has been carried out to compare both numerical models. Further case-studies need to be presented to compare the numerical results to an experimental structural behaviour. They will prove the importance of taking warping into account to represent the real behaviour of a structure.

Using the element with warping is about two times more computationally demanding than the plain Timoshenko fibre beam element. Nevertheless, a computation at the structural scale is still possible with the enhanced element. For an efficient model, enhanced fibre beam elements should be associated with classical elements and used only where necessary. The warping degrees of freedom need to be condensed out to the global beam degrees of freedom to enable the connection to different beam elements.

\section{ACKNOWLEDGEMENTS}

The authors would like to thank the PIA-SINAPS@ project (Séisme et Installation Nucléaire: Améliorer et Pérenniser la Sureté), approved and funded by the National Agency of Research (ANR) following the RSNR 2012 call for projects on future investments post-Fukoshima. (SINAPS@ ANR-11-RSNR-0022).
Capdevielle, S., S. Grange, F. Dufour, \& C. Desprez (2016). A multifiber beam model coupling torsional warping and damage for reinforced concrete structures. European Journal of Environmental and Civil Engineering 20(8), 914-935.

Capdevielle, S., S. Grange, F. Dufour, \& C. Desprez (submitted 2017). A fiber beam element with warping due to shear and torsion for the non-linear computation of concrete beams under multiaxial loadings. submitted to Engineering Structures, under review.

CEA (1998). Camus international benchmark. experimental results. synthesis of the participant reports. Technical report, CEA Saclay, France.

De Biasio, M. (2014). Ground motion intensity measures for seismic probabilistic risk analysis. $\mathrm{Ph}$. D. thesis, Université de Grenoble.

Grange, S. (2015). Modèles multi-échelles et algorithmes pour les simulations dynamiques: application 'a la vulnérabilité sismique des structures. Habilitation à diriger des recherches, Université de Grenoble-Alpes.

Grange, S., P. Kotronis, \& J. Mazars (2008, December). Numerical modelling of the seismic behaviour of a 7-story building: NEES benchmark. Materials and Structures 42(10), 14331442.

Kotronis, P. \& S. Grange (2010). Simplified modelling strategies for reinforced concrete structures. European Journal of Environmental and Civil Engineering 14(6-7), 823-838.

Kotronis, P. \& J. Mazars (2005). Simplified modelling strategies to simulate the dynamic behaviour of r/c walls. Journal of Earthquake Engineering 9(2), 285-306.

Le Corvec, V. (2012). Nonlinear $3 d$ frame element with multiaxial coupling under consideration of local effects. Ph. D. thesis, University of California, Berkeley.

Le Corvec, V. \& F. Filippou (2011). Enhanced 3d fiber beamcolumn element with warping displacements. In Proceedings of the 3rd ECCOMAS Thematic Conference on Computational Methods in Structural Dynamics and Earthquake Engineering.

Mazars, J., F. Hamon, \& S. Grange (2014). A new 3d damage model for concrete under monotonic, cyclic and dynamic loadings. Materials and Structures, 1-15.

Mazars, J., P. Kotronis, F. Ragueneau, \& G. Casaux (2006). Using multifiber beams to account for shear and torsion: Applications to concrete structural elements. Computer Methods in Applied Mechanics and Engineering 195(52), 7264-7281.

Menegotto, M. \& P. Pinto (1973). Method of analysis for cyclically loaded reinforced concrete plane frames including changes in geometry and non-elastic behavior of elements under combined normal force and bending. In IABSE Symposium on the Resistance and Ultimate Deformability of Structures Acted on by Well-Defined Repeated Loads, Lisbon.

Spacone, E. \& S. El-Tawil (2004). Nonlinear analysis of steelconcrete composite structures: State of the art. Journal of Structural Engineering 130(2), 159-168.

\section{REFERENCES}

Bairan, J. M. \& A. R. Mari (2006). Coupled model for the nonlinear analysis of anisotropic sections subjected to general $3 \mathrm{~d}$ loading. part 1: Theoretical formulation. Computers \& structures 84(31), 2254-2263.

Bairan, J. M. \& A. R. Mari (2007). Multiaxial-coupled analysis of rc cross-sections subjected to combined forces. Engineering structures 29(8), 1722-1738. 